\section{Chrischta Ganz}

Eine relativ junge Form der Phytotherapie ist die Gemmotherapie, die vor allem in Frankreich und Belgien bekannt ist, jedoch immer mehr auch in der Schweiz und in Deutschland Fuss fasst. Die Gemmotherapie ("Gemma» = lat. die Knospe) nutzt die grosse Vitalkraft und Lebendigkeit, die jede Knospe in sich trägt. Das Potenzial an Kraft und Entwicklung, wofür die Knospe (Abb. 1, 2) im konkreten wie auch im übertragenen Sinne steht, zeigt sich in der Heilund Regenerationskraft der Gemmotherapeutika [1].

Wer sich einen Pflanzentrieb vorstellt, der durch sein Streben nach Licht und Wachstum selbst dicken Asphalt durchbricht, sieht die Potenz der Meristeme bildlich vor sich. Hergestellt werden die Heilmittel aus dem Embryonalgewebe frischer Pflanzenknospen samt Vegetationspunkten, aus jungen Schösslingen, Wurzelfasern und Triebspitzen - also aus Gewebe, das mitten in der Vermehrung steht und somit reich an Wachstumsfaktoren wie Auxinen und Gibberellinen ist. Das höchste Potenzial an Vitalität einer Pflanze, im Embryonalgewebe beheimatet, wird durch die Gemmotherapeutika für den Menschen nutzbar.

Als Entdecker der Methode gilt Dr. Pol Henry aus Brüssel, der in den 1950er-Jahren seine Arbeiten unter dem Titel «Phytoembryopathie» veröffentlicht hat. Den heute gebräuchlichen Namen «Gemmotherapie» soll der französische Arzt Dr. Max Tétau eingeführt haben.

Grundlage der Arzneimittelherstellung ist seit 1965 die Pharmacopée française. Im Herbst 2011 wurde das Herstellungsverfahren für die Gemmotherapeutika neu in das euro-

\section{Fortbildung}

Schweiz Z Ganzheitsmed 2013;25:80-81

DOI: $10.1159 / 000348862$

\section{Gemmotherapie}

päische Arzneibuch (PhEur) aufgenommen.

Die Knospen, Wurzelfasern und Triebspitzen werden von Hand geerntet, meist aus Wildsammlung oder biologischem Anbau. In einer Alkohol-Glycerin-Lösung wird das Sammelgut mazeriert und so für den menschlichen Körper verfügbar gemacht. Da die Knospen einer Pflanze viele Aminosäuren und Proteine enthalten, sind klassische Auszugsmethoden mit Alkohol/Wasser nicht geeignet. Nach der Filtrierung werden die Mazerate im Verhältnis von eins zu zehn verdünnt. Höhere Verdünnungen sind nicht üblich.

Die Gemmotherapeutika werden meist als Spray verwendet, sodass die Knospenmazerate über die Mundschleimhaut aufgenommen werden können. Die Dosierung ist abhängig von Alter, Konstitution und Art der Erkrankung. Als generelle Empfehlung für Erwachsene gilt 3-mal zwei Sprühstösse und für Kinder 3-mal einen halben bis ganzen Sprühstoss.

Ursprünglich wurde empfohlen, die Mittel nur als Einzelmittel anzuwenden. Heutige Erfahrungen zeigen jedoch, dass die Mittel ebenso in Kombinationen (auch beispielsweise mit Spagyrika) wirksam sind.

\section{Wirkung der Gemmomazerate}

Die ersten wissenschaftlichen Untersuchungen in den 1950er-Jahren gehen auf Dr. Henry zurück. Heute untermauert eine Vielzahl pharmakologischer und analytischer Studien die ersten klinischen Resultate [2]. So ist beispielsweise in der Analyse der Inhaltstoffe in den verschiedenen Entwicklungsphasen der Pflanze ne-

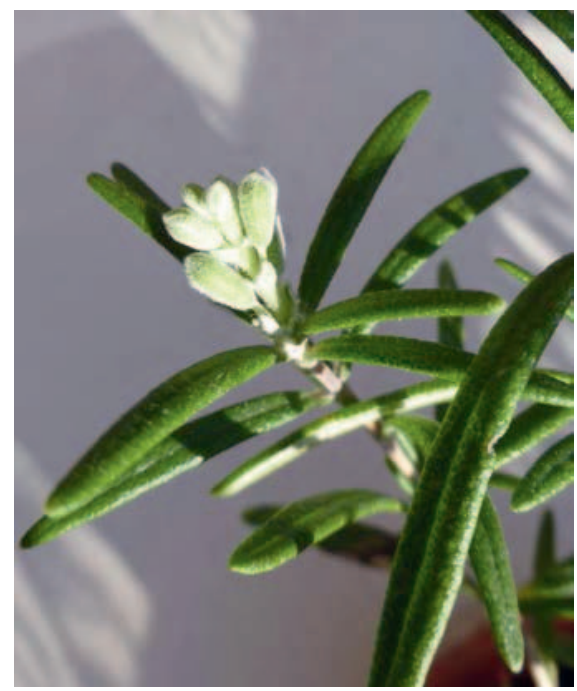

Abb. 1. Blütenknospe und junger Zweig des Rosmarinus officinalis.

ben einer deutlich grösseren Vielfalt an Aminosäuren auch deren wesentlich höherer Gehalt in den Knospen nachgewiesen.

Die Wirkung der Gemmomazerate basiert auf einer Monozyten-Makrophagen-Stimulation und somit auf einer Beeinflussung der Phagozytose. Die erhöhte Phagozytoseaktivität bewirkt eine stärkere Ausscheidung (mittels Elektrophorese nachweisbare Beeinflussung der Blutproteinkonzentrationen).

Die Knospenmazerate wirken somit reinigend, ausleitend und regulierend. Durch die Vitalkraft und Harmonisierung werden die körperlichen Heilungs- und Regenerationsprozesse gefördert. In der Praxis werden die Heilmittel bei akuten und chronischen Erkrankungen als eigenständige Therapie, als synergistisch wirkende Begleittherapie (zu einer homöopathischen, manuellen, energetischen oder konventionellen Behandlung) und auch in der Rekon-

\section{KARGER}

Fax +4976145207 14
(๑) 2013 S. Karger GmbH, Freiburg
Chrischta Ganz

Praxis für Naturheilkunde

Steinentischstrasse 1, 8002 Zürich, Schweiz

naturheilpraxis@chrischtaganz.ch 


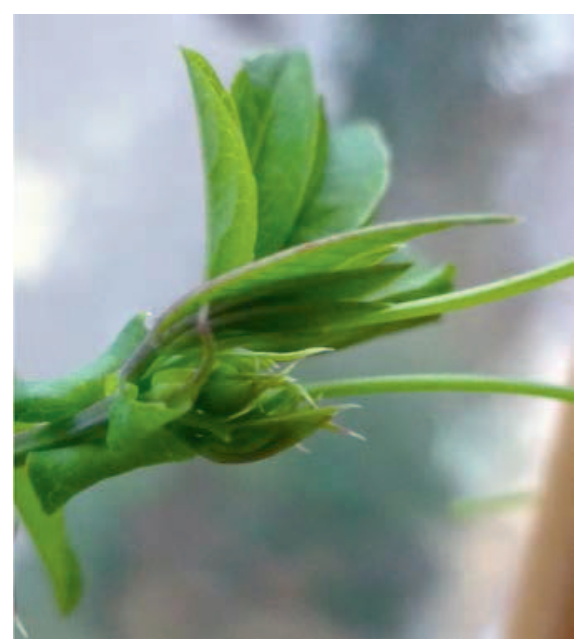

Abb. 2. Wer die Knospe einer Passiflora incarnata betrachtet und sich eine offene Passionsblume vorstellt, erkennt das Potenzial, das in einer Knospe schlummert.

valeszenz sowie zur Nachbehandlung nach Infektionen oder Antibiotikatherapie eingesetzt.

Die Knospenauszüge werden teilweise für ähnliche, bei einigen Pflanzen aber auch für andere Indikationen als die klassischen Phytotherapeutika eingesetzt. Gemmomazerate in guter Qualität sind von den Firmen Spagyros und Phytopharma erhältlich. Beide Hersteller bieten ausserdem sehr empfehlenswerte Weiterbildungen an.

\section{Eine Auswahl an Gemmomazeraten}

\section{Ribes nigrum}

(Schwarze Johannisbeere)

Es werden die frischen Knospen verwendet. Etwas salopp wird R. nigrum heute als «pflanzliches Cortison» bezeichnet, da die natürliche Synthese im Organismus unterstützt wird.

Wirkungen: Adaptogen, immunregulatorisch, entzündungshemmend (sowohl bei akuten wie auch chronischen Entzündungen [3]), antiallergisch, antidegenerativ, gefässprotektiv, analgetisch, endokrine Wirkung auf Nebenniere und Hypophyse, generell stärkend und aufbauend, blutbildend, ausserdem synergistische Wirkung auf die übrigen Knospen.

Indikationen: Allergien, Heuschnupfen, Ekzeme, Akne, Entzündungen des Bewegungsapparats, Entzündungen im Hals-Nasen-Ohren-Bereich, Bronchitis und Asthma, Prostatabeschwerden, Migräne, Immunschwäche, Rekonvaleszenz, Katabolismus.

\section{Rosa canina (Hagebutte)}

Die jungen, unverholzten Triebe werden verwendet. $R$. canina ist unter anderem das bewährte Mittel für Kinder, die «immer krank sind».

Wirkungen: Entzündungshemmend, immunstimulierend.

Indikationen: Hartnäckig rezidivierende Erkältungskrankheiten (Dauerschnupfen, Bronchitis, Rhinopharyngitiden, Otitis media etc.), Immunschwäche, Migräne, Haut- und Schleimhautentzündungen, Cor pulmonale, juvenile Wachstumsstörungen, Arthrose, Polyarthritis.

\section{Rosmarinus officinalis (Rosmarin)}

Hier werden ebenfalls die jungen, unverholzten Triebe verwendet.
Wirkungen: Cholagog, choleretisch, leberprotektiv, antioxidativ, stoffwechselregulierend, durchblutungsfördernd.

Indikationen: Ausleitung über die Leber, zur Bindung freier Radikale, Hypertriglyceridämie, Hypercholesterinämie, Hyperurikämie, periphere Durchblutungsstörungen, als Leberschutz (nach Hoefler et al. [4] 63\% höhere Leberschutzwirkung als Gesamtpflanze) (Cave: Hypertonie).

\section{Juniperus communis (Wacholder)}

Die jungen, unverholzten Triebe werden verwendet.

Wirkungen: Leberprotektiv, leberzellregenerierend, entzündungshemmend, aquaretisch, diuretisch, stoffwechselanregend, entgiftend.

Indikationen: Chronische Hepatitis (insbesondere bei medikamenteninduzierter Hepatitis und alkoholischer Leberzirrhose), Hyperurikämie, Ödeme, chronische Zystitis, Nephritis und Nephropathie, als Stoffwechselregulans bei Allergien, als Adjuvans bei einer Gewichtsreduktion, generell zur Ausleitung über Leber und Niere (Cave: Schwangerschaft).

\section{Literatur}

1 Steingassner H-M: Gemmotherapie-Phytotherapie-Mineralientherapie. Wien, Maudrich, 2005.

2 Tétau M: Nouvelles cliniques de Gemmothérapie. Paris, Éditions Similia, 1987.

3 Tétau M: Ribes nigrum, ein natürliches pflanzliches Mittel gegen Entzündungen. Zentralstelle für Dokumentation, LPh Dolisos.

4 Hoefler C: Comparative choleretic and hepatoprotective properties of young sprouts and total plant extracts of Rosmarinus officinalis in rats. J Ethnopharmacol 1987;19:133-143. 